\title{
Candidaemia and a risk predictive model for overall mortality: a prospective multicentre study
}

\author{
C. Keighley ${ }^{1,2,3^{*}}$ D, S. C-A. Chen ${ }^{1,2,3}$, D. Marriott ${ }^{4}$, A. Pope ${ }^{5,6}$, B. Chapman, K. Kennedy ${ }^{8}$, N. Bak ${ }^{9}$, N. Underwood ${ }^{10}$, \\ H. L. Wilson ${ }^{8}$, K. McDonald ${ }^{4}$, J. Darvall ${ }^{11}$, C. Halliday ${ }^{1}$, S. Kidd ${ }^{12}$, Q. Nguyen ${ }^{13}$, K. Hajkowicz ${ }^{14}$, T. C. Sorrell ${ }^{2,3,7}$, \\ S. Van $\mathrm{Hal}^{15}$ and M. A. Slavin ${ }^{16}$
}

\begin{abstract}
Background: Candidaemia is associated with high mortality. Variables associated with mortality have been published previously, but not developed into a risk predictive model for mortality. We sought to describe the current epidemiology of candidaemia in Australia, analyse predictors of 30-day all-cause mortality, and develop and validate a mortality risk predictive model.

Methods: Adults with candidaemia were studied prospectively over 12 months at eight institutions. Clinical and laboratory variables at time of blood culture-positivity were subject to multivariate analysis for association with 30-day all-cause mortality. A predictive score for mortality was examined by area under receiver operator characteristic curves and a historical data set was used for validation.

Results: The median age of 133 patients with candidaemia was 62 years; 76 (57\%) were male and 57 (43\%) were female. Co-morbidities included underlying haematologic malignancy $(n=20 ; 15 \%)$, and solid organ malignancy in $(n=25 ; 19 \%) ; 55$ (41\%) were in an intensive care unit (ICU). Non-albicans Candida spp. accounted for $61 \%$ of cases (81/133). All-cause 30-day mortality was 31\%. A gastrointestinal or unknown source was associated with higher overall mortality than an intravascular or urologic source $(p<0.01)$. A risk predictive score based on age $>65$ years, ICU admission, chronic organ dysfunction, preceding surgery within 30 days, haematological malignancy, source of candidaemia and antibiotic therapy for $\geq 10$ days stratified patients into $<20 \%$ or $\geq 20 \%$ predicted mortality. The model retained accuracy when validated against a historical dataset $(n=741)$.
\end{abstract}

Conclusions: Mortality in patients with candidaemia remains high. A simple mortality risk predictive score stratifying patients with candidaemia into $<20 \%$ and $\geq 20 \% 30$-day mortality is presented. This model uses information available at time of candidaemia diagnosis is easy to incorporate into decision support systems. Further validation of this model is warranted.

Keywords: Candidaemia, Candida blood stream infection, Mortality, Risk stratification score, Invasive fungal infection

\footnotetext{
* Correspondence: Caitlin.keighley@sydney.edu.au

${ }^{1}$ Centre for Infectious Diseases and Microbiology Laboratory Services, ICPMR,

New South Wales Health Pathology, Westmead Hospital, Darcy Rd, 3rd Level,

ICPMR Building, Westmead, Sydney, New South Wales 2145, Australia

${ }^{2}$ Marie Bashir Institute for Infectious Diseases and Biosecurity, The University

of Sydney, Sydney, NSW, Australia

Full list of author information is available at the end of the article
}

(c) The Author(s). 2019 Open Access This article is distributed under the terms of the Creative Commons Attribution 4.0 International License (http://creativecommons.org/licenses/by/4.0/), which permits unrestricted use, distribution, and

reproduction in any medium, provided you give appropriate credit to the original author(s) and the source, provide a link to the Creative Commons license, and indicate if changes were made. The Creative Commons Public Domain Dedication waiver (http://creativecommons.org/publicdomain/zero/1.0/) applies to the data made available in this article, unless otherwise stated. 


\section{Background}

Candidaemia (or bloodstream infection with Candida spp.) continues to increase and ranks amongst the top 5 hospital-acquired infections in many countries [1-5]. The shift in aetiology of candidaemia towards non-albicans Candida spp. and in particular, the disproportionate increase of Candida glabrata complex infections is of concern [2, 4]. Resistance to azole and echinocandin antifungal drugs, though increasing in certain regions, is uncommon in Australia [1]. Mortality from candidaemia remains high (up to 40\%) with prolonged hospital stay and excess costs [1-3, 5-7].

Better understanding of the variables that influence mortality is essential to improving outcomes in patients with candidaemia. A number of studies have found that mortality increases with age (e.g. > 65 years), admission to an intensive care unit (ICU), use of total parenteral nutrition (TPN) or broad-spectrum antibiotics, organ dysfunction and a gastrointestinal source of candidaemia [6, 8-11]. In addition, delays in source control and initiation of appropriate antifungal therapy adversely affect outcomes [12, 13].

Despite a number of models using risk stratification to predict the likelihood of developing candidaemia [11, 14 , to our knowledge, there is no similar stratification model to predict mortality. Stratification of the risk of mortality may help delineate patients in whom an aggressive approach to source control is needed, or, given that therapy with echinocandins requires intravenous therapy and hospitalisation, may guide an earlier transition to oral therapy $[15,16]$.

We conducted a contemporary multicentre, prospective study of the epidemiology and complications of candidaemia in Australia and assessed factors influencing mortality. Based on these data we propose a simple risk prediction model for overall mortality using clinical and laboratory variables known at the time of notification of a positive blood culture.

\section{Methods}

\section{Study design and data collection}

The study was a prospective observational study at eight tertiary referral hospitals carried out between March 2014 and February 2016. Patients were identified through active laboratory-based surveillance at each centre over a total of 12 months from time of commencement. Human research ethics approval was obtained with study oversight through the Western Sydney Local Health District HREC (HREC Ref: AU RED LNR14/WMEAD/112).

All adults $\geq 18$ years, with at least one blood culture positive for Candida spp., were enrolled. Episodes of recurrent candidaemia (occurring more than 30 days after the initial episode) within the study period were excluded. Cardiac echocardiography and an ophthalmology assessment were performed as directed by the treating clinician. Data collected on standardised case report forms included: patient demographics, healthcare setting, patient co-morbidities (e.g. malignancy, diabetes mellitus; Additional file 1), presence of a predisposing factor in the preceding 30 days (e.g. surgery and type thereof, central vascular access device [CVAD]), likely source of candidaemia (see Definitions below and in Additional file 1), complications, results of laboratory studies, and treatment and clinical outcomes at 30 days. All data were collected at baseline with progress including treatment and outcome at day 7 and 30 after the date of the initial positive blood culture or at death if this occurred earlier. Mortality was defined as due to candidaemia unless another identifiable cause was ascribed as per the treating physician. Periodic audits ensured complete case capture.

\section{Definitions}

A case (or episode) was defined as isolation of one or more Candida spp. from blood during the study period. The date of candidaemia was the date of first positive blood culture. Inpatient healthcare associated (IHCA) candidaemia were defined as episodes that occurred $>/=$ $48 \mathrm{~h}$ after hospital admission and which were not clinically manifest on admission. Among cases classed as outpatient-acquired candidaemia, episodes associated with recent healthcare contact events e.g. surgical procedures, were classed as outpatient healthcare associated episodes (OHCA) whilst cases with no healthcare -related risk factors were community-acquired (CA) [6]. The source of candidaemia was determined by the attending physician as i) intravascular (same Candida species isolated from the tip of an intravascular device as that from blood culture, or physician-ascribed where indwelling intravascular device was the only likely source, or candidaemia in the context of documented intravenous drug use as the only likely source); ii) gastrointestinal (same Candida species identified from specimens originating from a gastrointestinal or intra-abdominal source as that from blood culture or physician-ascribed where documented interventional procedure e.g. gastrointestinal surgery, biliary tract manipulation and no other likely source); iii) urologic (same Candida species isolated from urine as that from blood culture following antecedent urologic instrumentation or surgery that clearly preceded candidaemia and no other likely source); or iv) unknown (not attributable to an intravascular, gastrointestinal or urologic source) (see table included in Additional file 1). Sepsis at the time of blood culture collection was defined according to the Australian national sepsis guidelines [17]. Endocarditis 
was classified by modified Duke criteria [18]. Neutropenia was defined as a neutrophil count of $<1.0$ X $10^{9}$ cells/L.

\section{Microbiological methods}

Blood was cultured in BACTEC (Becton Dickinson, Sparks, MD, USA) or BacT/Alert 3D (bioMérieux, Marcy l'Etoile, France) blood culture automated systems. Candida organisms were identified to species level by matrix-assisted laser desorption/ionization- time of flight mass spectrosocopy (MALDI-TOF MS) (Biotyper database v 3.1; Bruker Daltoniks, Germany) or by Vitek 2 YST, 20C AUX or ID 32C identification systems (bioMérieux, Marcy-L'Étoile, France). All isolates were forwarded to a mycology reference laboratory (Westmead Hospital, Sydney, New South Wales or SA Pathology, Adelaide, South Australia) for species confirmation by internal transcribed spacer (ITS) sequencing [19] and for antifungal susceptibility testing [20,21]. Where species identification was discordant the reference laboratory determination was used.

\section{Statistical analysis}

Data were analyzed with $\mathrm{R}$ version 2.15 .13 ( $\mathrm{R}$ Core Team, Vienna, Austria). For univariate analysis, continuous variables were compared with the student $t$ test, and categorical variables compared with the two-tailed Chi-squared test with Yates continuity correction as indicated. A $p$ value $<0.05$ was considered significant. Odds ratios and 95\% confidence intervals (CI) were calculated. Kaplan-Meier and log-rank test analyses were used to test for an association between the source of candidaemia and mortality.

In order to produce a model which could be used to guide further treatment, input variables were restricted to those known at the time of positive blood culture. Variables with $p<0.15$ for death at 30 days on univariate analysis were included in the multivariate logistic regression model. For simplicity of use in hospital wards, all variables, including source of candidaemia, were converted to binary variables. In order to reduce the effect of possible over-fitting (otherwise known as optimism bias) multiple random subsets consisting of $80 \%$ of the sample were used to construct prediction models. These models were refined by a stepwise elimination procedure and then tested on the remaining $20 \%$ of the sample to evaluate performance. Variables which were important in a large proportion of these random test samples were retained in the final model. The final model was then tested against a historical national dataset of 741 prospectively recorded cases (2001-2004) [6].

\section{Results}

\section{Patient demographics}

There were 138 incident cases of candidaemia (96\%) of which 133 were evaluable (in five patients, data to 30 days were incomplete). The median patient age was 62 years (interquartile range, IQR 51-73); 76 (57\%) were male and $57(43 \%)$ were female (see Table 1). The proportion of patients with IHCA, OHCA and CA candidaemia is shown in Table 1 . For 55 patients (41\%) in ICU at the time of diagnosis, the median APACHE (Acute physiology and chronic health evaluation) II score for ICU patients was 19 (IQR 16-27) and the median length of ICU stay was 7 (IQR 2-14) days.

\section{Patient characteristics}

Summary statistics for demographic and risk factors in the cohort are presented in Table 1, as are the major co-morbidities and predisposing factors for candidaemia in the patient cohort.

There were 53 patients with two or more co-morbidities (40\%) and 35 patients (25\%) with none. The most common co-morbidity was diabetes mellitus (33 patients, 25\%) followed by cardiovascular disease (26 patients, 20\%) and solid organ malignancy (25 patients, 19\%) (Table 1). Of patients with solid organ malignancy, 7 were in ICU at the time of candidaemia diagnosis. Three had received cytotoxic chemotherapy and 18 had undergone surgery within the past 30 days. Of 20 patients with a haematological malignancy, 12 were neutropenic. Two patients had received an allogeneic stem cell transplant (SCT); one had received fluconazole for 21 days at the time of $C$. krusei candidaemia and the other had received liposomal amphotericin for more than 30 days at the time of $C$. albicans candidaemia.

Common predisposing factors included a central venous access device (CVAD; $n=98,74 \%$ ) and a urinary catheter $(n=80,60 \%)$. CVADs had been in situ for a median of 8 (IQR 3-17) days with 13 (13\%) in place for $>30$ days. Urinary catheters had been in situ for a median of 6 (IQR 2-15) days with 10 (13\%) in place for > 30 days. Hyperalimentation with TPN was administered to 27 patients $(20 \%)$, for a median 13 (IQR 5-20) days. There were 110 patients with at least 2 predisposing factors $(83 \%)$ and seven patients $(5 \%)$ with none. The latter included two patients with neither a co-morbidity nor a predisposing factor.

Antibacterial drugs were administered to 119 patients (89\%) for a median of 5 (IQR 1-11) days prior to diagnosis of candidaemia and $58 \%$ received two or more antibiotics (Table 1). There was no association between any particular antibiotic and Candida spp. on univariate or multivariate analysis. Twenty patients developed candidaemia (Table 1) whilst receiving a prophylactic antifungal agent (median 7 days [IQR 3-16]). In 16/20 
Table 1 Characteristics of patients with candidaemia and univariate analysis for all cause 30-day mortality

\begin{tabular}{|c|c|c|c|c|}
\hline & No. patients (total $=133$ ) & 30-day mortality & Odds ratio, $95 \%$ confidence interval & $P$ value \\
\hline Age $>65$ years & $57(43)$ & $24(42)$ & $2.7(1.3-5.8)$ & 0.01 \\
\hline Male gender & $76(57)$ & $24(32)$ & $1.1(0.5-2.3)$ & 0.8 \\
\hline Female gender & $57(43)$ & $17(30)$ & $0.9(0.4-1.9)$ & 0.8 \\
\hline \multicolumn{5}{|l|}{ Setting of candidaemia } \\
\hline IHCA & $113(85)$ & $35(31)$ & $1.0(0.4-3.0)$ & 0.9 \\
\hline $\mathrm{OHCA}$ & $13(10)$ & $3(23)$ & $0.6(0.2-2.5)$ & 0.6 \\
\hline CA & $7(5)$ & $3(43)$ & $0.6(0.2-2.5)$ & 0.5 \\
\hline \multicolumn{5}{|l|}{ Admitting service } \\
\hline Medical & $77(58)$ & $30(39)$ & $2.6(1.2-5.8)$ & 0.02 \\
\hline Surgical & $56(42)$ & $11(20)$ & $0.4(0.2-0.8)$ & 0.02 \\
\hline ICU admission & $55(41)$ & $22(40)$ & $2.1(1.0-4.4)$ & 0.06 \\
\hline \multicolumn{5}{|l|}{ Co-morbidities } \\
\hline Haematologic malignancy & $20(15)$ & $10(50)$ & $2.6(1.0-7.0)$ & 0.06 \\
\hline Allogeneic stem cell transplantation & $2(1)$ & $1(50)$ & $2.3(0.1-37.3)$ & 0.6 \\
\hline Solid organ malignancy & $25(19)$ & $7(28)$ & $0.8(0.3-2.2)$ & 0.7 \\
\hline Solid organ transplantation & $5(4)$ & $1(20)$ & $0.5(0.1-5.0)$ & 0.6 \\
\hline Diabetes mellitus & $33(25)$ & $10(30)$ & $1.0(0.4-2.3)$ & 0.9 \\
\hline Chronic organ dysfunction & $50(38)$ & $29(58)$ & $0.4(0.2-0.9)$ & 0.03 \\
\hline Renal disease & $18(14)$ & $8(44)$ & $2.0(0.7-5.5)$ & 0.2 \\
\hline Liver disease & $15(11)$ & $5(33)$ & $1.1(0.4-3.6)$ & 0.8 \\
\hline Cardiovascular disease & $26(20)$ & $11(42)$ & $1.8(0.8-4.6)$ & 0.2 \\
\hline Respiratory disease & $11(8)$ & $5(45)$ & $2.0(0.6-6.9)$ & 0.3 \\
\hline \multicolumn{5}{|l|}{ Predisposing factors } \\
\hline Surgery & $69(52)$ & $16(23)$ & $0.5(0.2-1.0)$ & 0.05 \\
\hline Gastrointestinal surgery & $35(26)$ & $11(31)$ & $1.0(0.4-2.4)$ & 0.9 \\
\hline Urinary catheter & $80(60)$ & $15(19)$ & $1.6(0.7-3.6)$ & 0.2 \\
\hline CVAD & $98(74)$ & $30(31)$ & $1.0(0.4-2.2)$ & 0.9 \\
\hline Hyperalimentation & $27(20)$ & $5(19)$ & $0.4(0.2-1.3)$ & 0.1 \\
\hline Corticosteroids or other immunosuppressant & $36(27)$ & $13(36)$ & $1.4(0.6-3.1)$ & 0.4 \\
\hline Intravenous drug use & $13(10)$ & $1(8)$ & $0.2(0.02-1.3)$ & 0.1 \\
\hline Use of antibiotic agents $\geq 10$ days & $39(29)$ & $16(41)$ & $1.9(0.9-4.2)$ & 0.1 \\
\hline Prior antifungal use $\mathrm{e}^{\mathrm{a}}$ & $19(14)$ & $7(37)$ & $1.4(0.5-3.8)$ & 0.5 \\
\hline Sepsis syndrome & $97(73)$ & $29(30)$ & $0.9(0.4-1.9)$ & 0.5 \\
\hline \multicolumn{5}{|l|}{ Source of candidaemia } \\
\hline Intravascular & $42(32)$ & $9(21)$ & $0.5(0.2-1.2)$ & 0.1 \\
\hline Gastrointestinal & $46(35)$ & $19(41)$ & $2.1(1.0-4.4)$ & 0.06 \\
\hline Urologic & $27(20)$ & $2(7)$ & $0.1(0.03-0.6)$ & 0.009 \\
\hline Unknown & $18(14)$ & $11(61)$ & $4.4(1.6-12.5)$ & 0.005 \\
\hline \multicolumn{5}{|l|}{ Candida species } \\
\hline Candida albicans & $52(39)$ & $17(33)$ & $1.2(0.5-2.4)$ & 0.7 \\
\hline Candida glabrata complex & $43(32)$ & $15(35)$ & $1.4(0.5-3.8$ & 0.5 \\
\hline Candida parapsilosis complex & $13(10)$ & $1(8)$ & $0.2(0.02-1.3)$ & 0.09 \\
\hline Other Candida species ${ }^{b}$ & $25(19)$ & $8(32)$ & $1.1(0.4-2.7)$ & 0.9 \\
\hline
\end{tabular}

Counts are shown as $\mathrm{n}(\%)$ unless otherwise stated

CA community acquired, CVAD Central venous access device, IHCA inpatient healthcare associated, ICU intensive care unit, OHCA outpatient healthcare associated a Antifungals used prior to diagnosis of candidaemia included fluconazole in 15 patients, voriconazole in 1, anidulafungin in 1, caspofungin as prophylaxis in 2 and voriconazole for treatment of possible pulmonary aspergillosis in 1

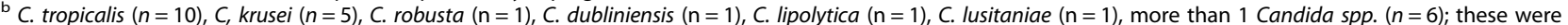
C. albicans and C. glabrata sensu stricto $(n=2), C$. albicans and C. bracariensis $(n=1)$, C. albicans and C. nivariensis $(n=1), C$. albicans and C. parapsilosis sensu stricto $(n-1)$, and C. albicans, C. parapsilosis sensu stricto and C. tropicalis $(n=1)$ 
patients, the Candida species was susceptible or wild-type to the prior antifungal agent used.

\section{Microbiology}

Candida albicans caused 52 (39\%) episodes followed by C. glabrata complex (43, 32\%) and C. parapsilosis complex (13, 10\%) (Table 1). Non-albicans Candida spp. predominated; non-albicans Candida spp. accounted for $58 \%(65 / 112)$ of IHCA, 84\% (11/13) of OHCA and $63 \%$ $(5 / 8)$ of CA episodes. Isolation of the C. glabrata complex was not associated with age $>65$ years, or with particular co-morbidities or predisposing factors (data not shown). Prior antifungal use $(n=20)$ did not correlate with Candida spp. identified in this context.

C. glabrata complex was isolated significantly more frequently from patients with a probable gastrointestinal source than alternative sources $(20 / 46,43 \%$ vs. $23 / 87$, 26\%; OR 2.14 (95\%CI 1.01-4.54); $p=0.05)$. Conversely, C. parapsilosis complex was more common in candidaemia associated with an intravascular source compared with a source classed as "non-intravascular" (9/42, 21\% vs. 4/91, 44\%; OR 5.93 (95\%CI 1.71-20.58; $p=0.002)$. Recovery of $C$. albicans was equally likely regardless of attributable source $(16 / 42,38 \%$ intravascular, $15 / 46,33 \%$ gastrointestinal, $12 / 27,44 \%$ renal tract, 9/18, 50\% unknown).

\section{Complications}

Complications of candidaemia were documented in $10 \%$ $(13 / 133)$ of episodes. Endocarditis was identified in 5\% $(6 / 110)$ of patients undergoing echocardiography and endophthalmitis in $6 \%(6 / 98)$ of patients undergoing ophthalmological assessment. One patient had hepatosplenic candidiasis. There were no cases of fungal meningitis. Mortality at 30 days for patients with these complications was 5/13 (38\%).

\section{Therapy}

Amongst 117 patients who received antifungal therapy at diagnosis of candidaemia, 54 (46\%) were commenced on fluconazole and 60 (51\%) an echinocandin at doses concordant with published guidelines [15]. Sixteen patients (12\%) received only palliative therapy (with no antifungals) or died prior to treatment. Seventy-eight of 133 (59\%) episodes were due to isolates which were susceptible to fluconazole and of the patients who received fluconazole as empiric therapy, $46 / 54$ (85\%) of isolates were susceptible to fluconazole. All isolates were susceptible to echinocandins. The median duration of antifungal therapy for uncomplicated candidaemia was 15 days (IQR 14-22 days).

The 30-day mortality in patients who received fluconazole $(10 / 54,19 \%)$ vs an echinocandin $(14 / 60,23 \%)$ was similar (data not shown; $p=0.5$ ), after adjusting for variables identified in our mortality risk predictive score and Candida species. Only two of the patient deaths occurring in the initial fluconazole-treated group was associated with non-susceptibility to fluconazole.

Nineteen patients who received an echinocandin as initial antifungal therapy were stepped down to fluconazole after a median of 4 days (IQR 2-5); 11 (58\%) patients had therapy de-escalated after 4 days or less (9 after identification of Candida species and 2 after susceptibility results). There was no correlation between shorter time to step-down and outcome.

\section{Outcome}

The 7-day overall mortality was 21\% (28/133), and 30-day mortality, 31\% (41/133) with a candidaemia-attributed mortality of $13 \%(17 / 133)$ as per treating physician. At 30 days, the condition of only 66 patients (50\%) was either resolved or improved as judged by their physician. Mortality was lower in patients with an intravascular or urologic source of candidaemia than in patients with a gastrointestinal or unknown source $(p<0.01)$ (Fig. 1). CVADs had been removed within $72 \mathrm{~h}$ of the diagnosis of candidaemia in $87 \%$ (85/98); time to removal of CVAD was not significantly correlated with mortality. Sepsis was present in $73 \%(97 / 133)$ and was not correlated with mortality (Table 1).

\section{Mortality risk predictive score}

The univariate analysis is summarised in Table 1. By multivariate logistic regression analysis, mortality was associated with age $>65$ years, presence in ICU at the time of diagnosis of candidaemia, a haematological malignancy, organ failure within the prior 30 days, absence of recent surgery, receipt of antibiotics for $\geq 10$ days at diagnosis of candidaemia and an abdominal or unknown source of candidaemia (Table 2, Additional file 1). The APACHE II score was not included as a variable in the risk predictive score as it was only available for patients admitted to ICU. For in-sample prediction the AUC was 0.81. The score was out of a maximum of 7.5 points with higher points equating to higher mortality. All coefficients were close to 1 or 1.5 , therefore, to produce a tool that could be calculated with minimum difficulty on the ward, the coefficients were adjusted to produce the Score column in Table 2. Using these approximate coefficients in the final logistic regression model gave predictions close to those obtained using the original coefficients (AUC 0.80 , see Fig. 2). A score of 0 had a negative predictive value (NPV) of 100\%; there were 0 deaths amongst 10 patients in the current dataset. 


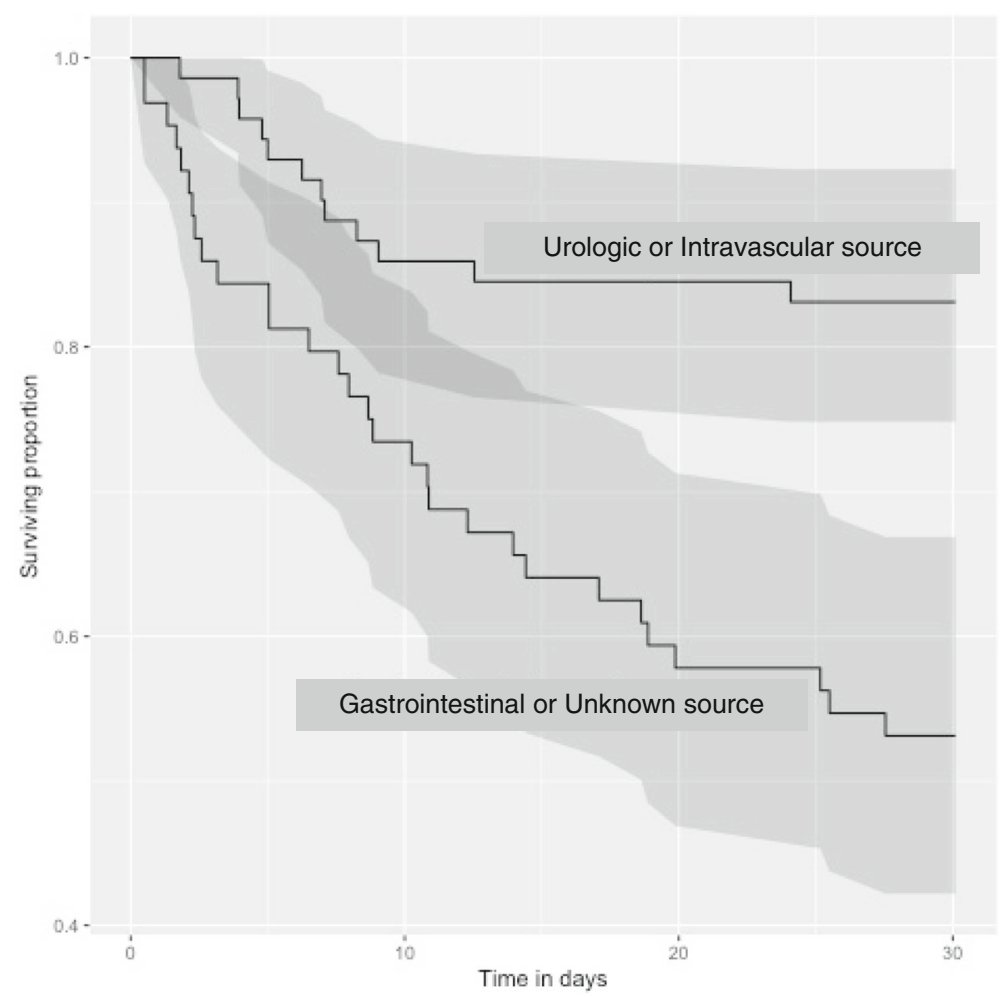

Fig. 1 Kaplan-Meier survival curve of all patients with candidaemia stratified by source; genitourinary/intravascular or gastrointestinal/unknown. Source was grouped to produce a binary variable of favourable versus unfavourable outcome. Log-rank test statistic for equality of curves: chisquared $=6.7$ on 1 degrees of freedom, $p=0.00967$. Shaded areas are pointwise $95 \%$ confidence intervals, showing the curves are well-separated

In order to produce a binary prediction for each individual, risk predictive scores were grouped by assigning individuals with risk predictive scores in the range 0 to 2 inclusive to the "lower-risk" category. According to the model, mortality in this group was less than $20 \%$ at 30 days. The threshold of 2 was chosen because this was a natural break in the data: patients with risk predictive scores greater than this had at least 33\% mortality at 30 days, according to the model. There was little change in the Positive Predictive Value (PPV) over the risk predictive score range 1 to 2 (Table 3 ).

\section{Validation of mortality risk predictive score}

Testing on an independent historical dataset [6] of 741 cases of candidaemia gave an AUC of 0.74 . The historical validation data set comprised $28 \%$ (216/741) patients in ICU and 48\% (356/741) had C. albicans candidaemia. The data retained an accurate split in predicted mortality at a score of 2; mortality in patients with a score greater than 2 had a 30-day all-cause mortality of at least $25 \%$. A score of 0 predicted a group with $<5 \%$ mortality; there was 1 death amongst 32 patients (3.1\%) in the historical dataset (Table 3).

Table 2 Risk prediction model for all cause 30-day mortality

\begin{tabular}{|c|c|c|c|c|c|}
\hline & Coefficient & SE & OR & $95 \% \mathrm{Cl}$ of $\mathrm{OR}$ & Score \\
\hline Age $>65$ years & 1.4 & 0.5 & 3.8 & $1.6-10.0$ & 1.5 \\
\hline Location in ICU at time of diagnosis & 0.9 & 0.5 & 2.5 & $1.0-6.4$ & 1 \\
\hline No prior surgery & 1.0 & 0.5 & 2.6 & $1.0-6.9$ & 1 \\
\hline Any haematological malignancy & 1.0 & 0.6 & 2.8 & $0.85-9.4$ & 1 \\
\hline Chronic organ dysfunction $^{a}$ & 1.0 & 0.5 & 2.7 & $1.1-6.6$ & 1 \\
\hline Gastrointestinal or unidentified attributable source & 0.9 & 0.4 & 2.5 & $1.1-6.1$ & 1 \\
\hline Use of antibiotic agents $\geq 10$ days & 1.0 & 0.5 & 2.6 & $1.0-6.7$ & 1 \\
\hline
\end{tabular}

${ }^{a}$ One or more of renal disease, liver disease, cardiovascular disease or respiratory disease (Additional file 1) SE Standard error of coefficient, OR Odds ratio, $\mathrm{Cl}$ Confidence interval

As all coefficients were close to 1 or 1.5 , the coefficients were adjusted to produce the Score column 


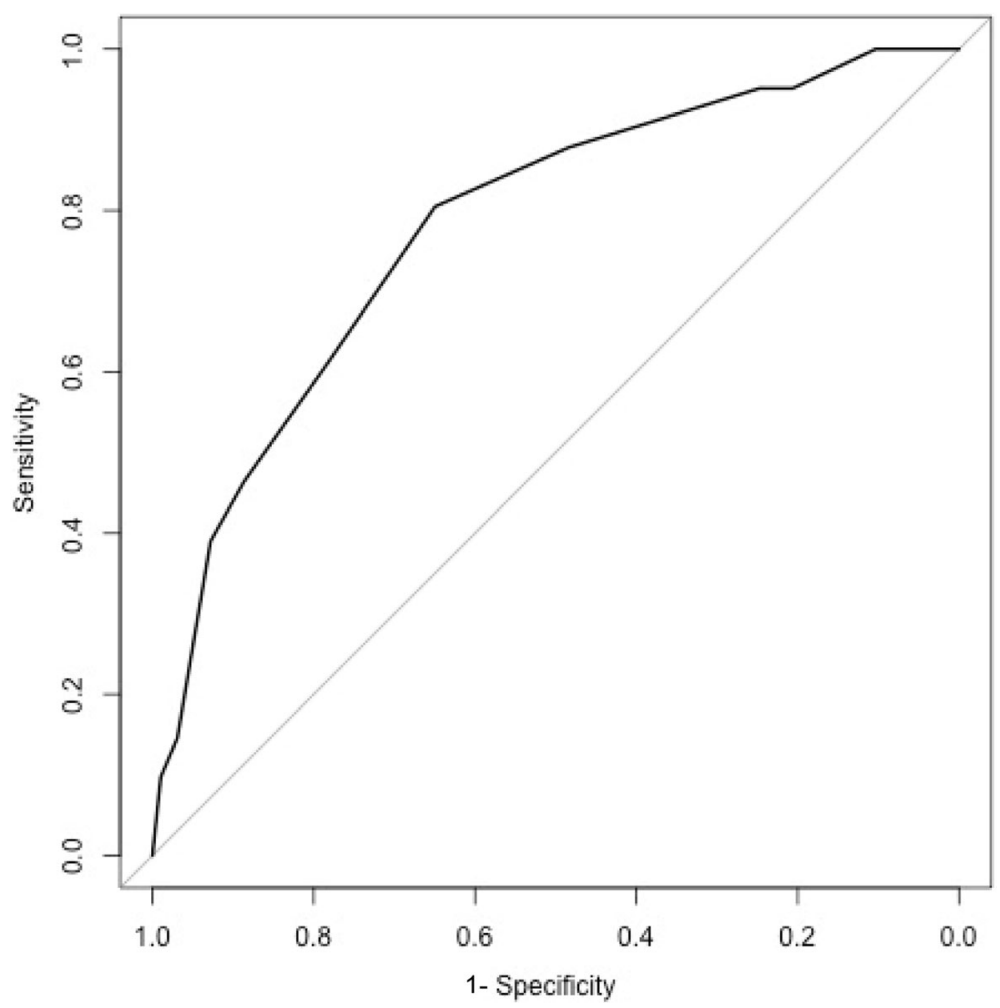

Fig. 2 Area under the curve (AUC) $=0.81$ for derivation cohort. When applied to $n=741$ historical cohort data (2001-2004), AUC =0.74

\section{Discussion}

Overall mortality in patients with candidaemia has not improved in the past 20 years despite the introduction of echinocandins, either in Australia, or internationally $[5,6,22]$. In the present study, we present the first data on a risk stratification tool for all-cause mortality in candidaemia. Notably, $41 \%$ of patients in the derivation and $29 \%$ in the validation cohort were in ICU, suggesting that the model can be generalised to non-critical care settings. A score of $>2$; constituting either age $>65$ years and at least one score criterion, or $\leq$ 65 years and more than two criteria from the score, identified a group with high mortality of at least $20 \%$ where an aggressive approach is warranted including source control and management of predisposing factors. Additionally, these patients may constitute a high-risk group for inclusion into clinical trials. Conversely a score of 0 was associated with a predicted and observed mortality of $<$ $5 \%$, though this applied to few patients.

The source of candidaemia was gastrointestinal or urologic in a majority (55\%) of cases in contrast with previous reports from our group and others, where CVAD-related candidaemia predominated [6, 23]. The urinary tract may be an increasing source of candidaemia [24-26]. Urological interventions such as those aimed to remove stones or stents may induce

Table 3 Performance of the mortality prediction model for individual score values

\begin{tabular}{|c|c|c|c|c|c|c|c|c|c|c|c|c|}
\hline Score & 6.5 & 5.5 & 5 & 4.5 & 4 & 3.5 & 3 & 2.5 & 2 & 1.5 & 1 & 0 \\
\hline Cumulative fraction with value $\geq$ to Score & 0.7 & 5.2 & 8.2 & 17.9 & 23.1 & 36.6 & 53.0 & 63.4 & 82.1 & 85.1 & 92.5 & 100.0 \\
\hline PPV & 100 & 86 & 73 & 71 & 65 & 55 & 51 & 44 & 35 & 34 & 33 & 31 \\
\hline NPV & 69 & 70 & 72 & 73 & 78 & 80 & 84 & 92 & 92 & 92 & 90 & 100 \\
\hline Sens & 2 & 15 & 20 & 41 & 49 & 66 & 88 & 90 & 95 & 95 & 100 & 100 \\
\hline Spec & 100 & 100 & 99 & 97 & 92 & 88 & 76 & 62 & 48 & 24 & 19 & 11 \\
\hline
\end{tabular}

Data presented as \%

A cut off of 2 divided the current, derivation cohort into $<20 \%$ or $>20 \%$ mortality

Using a score $>2$ on the historical, validation dataset, 161 died out of 393 at risk, compared with an expected number of 223, whilst in those with scores $\leq 2,41$ died out of 349 at risk, compared with an expected number of 39

In those with a score of 0 in the historical, validation dataset, one death was predicted and observed. A score of 0 had a NPV of $100 \%$ with a predicted and observed mortality $<5 \%$ though this only incorporated $7.5 \%$ (10/133) of the current, derivation cohort and $4.3 \%$ of the historical (32/741) cohort 
candidaemia, as observed in our cohort. Since the last report where CVAD-related candidaemia was more common [6], national infection prevention programs have been implemented that target optimisation of hand hygiene and reduction of central line infections via the use of central line insertion bundles [17, 27, 28]. Although time to removal of CVADs did not influence outcome in our cohort, this differs from findings of a previous study where earlier removal of lines correlated with decreased mortality and likely reflects prompt removal upon diagnosis in the current study [10]. Whilst the management of CVADs has improved, the increase in candidaemia from a gastrointestinal source has been reported widely $[29,30]$. The finding that absence of recent surgery was associated with mortality may indicate that sources not amenable to surgical intervention did more poorly; data examining surgical intervention would help clarify this in future studies. Furthermore, a substantial proportion of cases from an "unknown source" is likely to originate from the gastrointestinal tract due to the phenomenon of bacterial translocation associated with critical illness [31]. A gastro-intestinal or unknown source was an important predictor of mortality, confirming that source is pivotal to outcome [9, 12].

Previous data have linked source with Candida species $[29,30,32]$ and in the present series the C. glabrata complex was associated with a gastrointestinal source; although not advanced age or co-morbidities, as has been reported by others [26, 29, 30]. In Australia, the proportion of candidaemia due to $C$. albicans has fallen further (39\%; compared to 47\% in 2001-2004 [6]) regardless of setting. Non-albicans Candida spp. predominated, reflecting a global trend $[4,5]$.

Complicated candidaemia (the presence of metastatic infection) was reported in $10 \%$ of episodes and rates of endocarditis and endophthalmitis were consistent with those reported previously $[6,15,23,33]$. As not all patients underwent echocardiography and ophthalmological assessment these are likely to be underestimated.

Mortality in patients treated with fluconazole versus an echinocandin as initial therapy was not significantly different. This may be particular to settings such as in Australia, with a low prevalence of azole resistance [1] and close attention to source control. Notably, all 10 deaths in 54 patients treated with fluconazole as initial therapy, occurred in patients with a mortality risk predictive score of $\geq 2.5$; this could not be attributed to therapy (standard dosing of fluconazole e.g. $400 \mathrm{mg}$ daily) or the isolates (8 of the respective isolates were susceptible to fluconazole with an $\mathrm{MIC}<1 \mathrm{mg} / \mathrm{L}$ (data not shown). A mortality prediction tool may highlight patients with a higher risk of death not otherwise recognised.
Sepsis [17] was present in the majority of patients and thus did not discriminate those at higher risk of death. The recently published sepsis definitions could be explored in future studies, however they are yet to come into mainstream use either locally or internationally, and perform less well outside of the ICU setting [34, 35]. The APACHE II score is complex, not routinely calculated for non-ICU patients, and as we aimed to develop a score that could be applied to "all-comers" with candidemia, was not included in the model. Additionally, in the subset of patients in ICU, APACHE II score did not perform as well as our derived mortality risk predictive score (data not shown). Location in ICU at the time of diagnosis independently predicted 30-day mortality and was incorporated into the score.

The mortality risk predictive score was derived from variables known at the time of candidaemia diagnosis. Despite differences between the data in this study and the historical cohort data used for validation of the mortality risk predictive score including a longer time to removal of CVAD and predominance of azole therapy [6], the score retained utility. A score of 0 predicted mortality $<5 \%$ though this applied only to $7.5 \%$ of the current cohort and $4.3 \%$ of the historical cohort. The dichotomous prediction model identified a cohort with $<20 \%$ mortality that incorporated $36.6 \%$ of the current cohort and $47 \%$ of the historical cohort.

Strengths of our study include the multicentre prospective data collection of all sequential adult patients with candidaemia, and validation of the derived mortality risk predictive score on an independent dataset of 741 patients. Limitations include restriction of data collection to large hospitals, limited sample size and validation on a historical dataset. Whilst our data identified prolonged antibiotic use as important in mortality, data regarding bacterial infection were not collected and further analyses including this would be useful. Beta-D glucan was unavailable and procalcitonin was not routinely used in the settings assessed; incorporation of these in future studies may be informative. Future prospective studies would also benefit from inclusion of more centres.

\section{Conclusions}

In conclusion, mortality in patients with candidaemia remains high and we present a novel, simple prediction model that may be applied at a clinically useful time point to stratify predicted mortality of patients with candidaemia. In the present study, non-albicans Candida spp. predominated and a gastrointestinal and urologic source of candidaemia were common, demonstrating contemporary changes in the demographics of patients with candidemia. 


\section{Additional file}

Additional file 1: Definitions used in the present study and their explanations. Definitions used in study [36]. (DOCX 18 kb)

\section{Abbreviations}

APACHE: Acute physiology and chronic health evaluation; CA: Community acquired; CVAD: Central venous access device; GVHD: Graft versus host disease; ICU: Intensive care unit; IHCA: Inpatient healthcare associated; IQR: Interquartile range; ITS: Internal transcribed spacer; MALDI-TOF MS: Matrix-assisted laser desorption/ionization-time of flight mass spectroscopy; OHCA: Outpatient healthcare associated; TPN: Total parenteral nutrition

\section{Acknowledgements}

This data was presented at the 20th Immunocompromised Host Symposium, Athens, Greece, (17-19 June 2018)

The authors thank Ms. Catherine Ferguson, Dr. Khai Lin Huang and Dr. Kathryn Daveson for their assistance with data collection.

We also acknowledge the contributors to the historical data set from Australian Candidaemia Study (2001-2004) [6] contributed to by: Queensland Cairns Base Hospital (J. McBride); Calboolture Hospital (C. Coulter); Mater Adult Hospital (J. McCormack, K. Walmsley); Princess Alexandra Hospital (D. Looke, B. Johnson, G. Nimmo, G. Playford); Queensland Medical Laboratories (D. Drummond, R. Forgan-Smith); Rockhampton Hospital (E. Preston); Royal Brisbane Hospital (A. Allworth, J. Faoagali, N. Gerns); Sullivan and Nicolaides Pathology (J. Botes, S. Cherian, J. Robson, R. Vohra); Townsville Hospital (R. Norton); The Prince Charles Hospital (C. Coulter, G. O'Kane); New South Wales: Albury Base Hospital (D. Robb); Concord Hospital (T. Gottlieb); Douglass Hanly Moir Pathology (I. Chambers); Gosford Hospital (D. DeWit); Griffith Hospital Base (M. Carroll); Hunter Area Pathology Service (P. Dobson, J. Ferguson, S. Graves, L. Tierney); Liverpool Hospital (F. Jozwiak, R. Munro, V. Tomasotos); Manning Base Hospital (R. Pickles); Mayne Health (J. Holland); Narrabri District Hospital (F. Groenwald); New Children's Hospital (K. Hale, M. Watson); Orange Base Hospital (R. Vaz); Prince of Wales Hospital (R. Hardiman, C. Baleriola, S. Ryan); Royal North Shore Hospital (R. Pritchard, K. Weeks); Royal Prince Alfred Hospital (R. Benn, N. Adams); St. George Hospital (R. Lawrence, P. Taylor); St. George Private Hospital (S. Lindstrom); St. Vincent's Private and St. Vincent's Public Hospital (J. Harkness, D. Marriott, Q. Nguyen); Sydney Children's Hospital (P. Palasanthrian); Sydney Adventist Hospital (R. Grant, R. McPetrie); Wagga Wagga Base Hospital (R. Johnson); Westmead Hospital (S. Chen, C. Halliday, K. Maszewska, O.C. Lee, W. Meyer, T. Sorrell); Wollongong Hospital (N. Dennis, P. Newton); Victoria: Alfred Hospital (C. Franklin, O. Morrisey, M. Slavin, D. Spelman, S. Wesselingh); Austin and Repatriation Hospital (B. Speed); Bendigo Health Care Group (J. Hellsten, B. Mayall, J. Russell); Frankston Hospital (S. Broughton, I. Woolley); Melbourne Pathology (S. Coloe); Melbourne Private Hospital (A. Sherman); Monash Medical Centre (T. Korman); PathCare Consulting Pathologists (S. Graves); Peter MacCallum Cancer Institute (M. Slavin, M. Huysmans); Royal Melbourne Hospital (M. Slavin, A. Sherman); South Australia: Flinders Medical Centre (D. Gordon); Royal Adelaide Hospital (K. Rowlands, D. Shaw, W. Ferguson); Women's and Children's Hospital (D. Ellis, B. Ritchie, R. Handke); Western Australia: Fremantle Hospital (M Beaman, A. Chiam, J. McCarthy); Royal Perth Hospital (C. Heath); Sir Charles Gairdner Hospital (S. Altmann, I. Arthur, D. Speers); Tasmania: Launceston General (E. Cox); Royal Hobart Hospital (L. Cooley, A. McGregor); Northern Territory: Royal Darwin Hospital (B. Currie, G. Lum, D. Fisher); Australian Capital Territory: The Canberra Hospital (P. Collignon, J. Roberts, A. Watson).

\section{Funding}

This work was supported in part by an educational grant from MSD Australia. MSD Australia had no role in the design of the study, data collection, analysis, interpretation of data or writing of the manuscript.

\section{Availability of data and materials}

The datasets used and analysed during the current study are available from the corresponding author on reasonable request.

\section{Authors' contributions}

Conceptualisation and study design: CK, SC, MS, DM, BC, CH, TS, SVH Investigation: CK, SC, MS, DM, BC, KK, NB, NU, HW, KM, JD, CH, SK, QN, KH, TS,
SVH. Project collaboration: CK, SC, MS, DM, BC, KK, NB, NU, HW, KM, JD, CH, SK, QN, KH, TS, SVH. Supervision: SC, MS, DM, SVH, TS. Data analysis: CK, MS, AP, BC. Writing - original draft: CK, MS, SC, AP. Writing - reviewing \& editing: CK, SC, DM, AP, KK, NB, HW, JD, CH, SK, KH, TS, SVH, MS. All authors read and approved the final manuscript.

\section{Ethics approval and consent to participate}

Human research ethics approval was obtained for all sites, with the need for consent waived as a Low and Negligible Risk (LNR) research project, through the Western Sydney Local Health District Human Research Ethics Committee (HREC) (HREC Ref: AU RED LNR14/WMEAD/112).

\section{Consent for publication}

Not applicable.

\section{Competing interests}

SC, MS, SVH and DM are on the Antifungal Advisory Boards of Gilead Sciences Inc., MSD Australia and Pfizer Australia. SK is on the Antifungal Advisory Board of Pfizer Australia, MSD Australia and Mayne Pharma. TS, DM, MS, SC, SVH, NB, CK and SK have received untied grants from Gilead Sciences Inc., MSD Australia and Pfizer Australia. All other authors: none to declare.

\section{Publisher's Note}

Springer Nature remains neutral with regard to jurisdictional claims in published maps and institutional affiliations.

\section{Author details}

${ }^{1}$ Centre for Infectious Diseases and Microbiology Laboratory Services, ICPMR, New South Wales Health Pathology, Westmead Hospital, Darcy Rd, 3rd Level, ICPMR Building, Westmead, Sydney, New South Wales 2145, Australia. ${ }^{2}$ Marie Bashir Institute for Infectious Diseases and Biosecurity, The University of Sydney, Sydney, NSW, Australia. ${ }^{3}$ Department of Infectious Diseases, Westmead Hospital, Westmead, Sydney, NSW, Australia. ${ }^{4}$ Department of Microbiology and Infectious Diseases, St. Vincent's Hospital, Sydney, NSW, Australia. ${ }^{5}$ Eastern Health Clinical School, Monash University, Melbourne, Victoria, Australia. ${ }^{6}$ School of Mathematics and Statistics, University of NSW, Sydney, NSW, Australia. ${ }^{7}$ Westmead Institute for Medical Research, Westmead, NSW, Australia. ${ }^{8}$ Department of Infectious Diseases and Microbiology, Canberra Hospital, Australian National University Medical School, Canberra, ACT, Australia. 'Department of Infectious Diseases, Royal Adelaide Hospital, Adelaide, SA, Australia. ${ }^{10}$ Infection Management Services, Princess Alexandra Hospital, Brisbane, QLD, Australia. ${ }^{11}$ Department of Intensive Care, Royal Melbourne Hospital, Melbourne, VIC, Australia.

${ }^{12}$ National Mycology Reference Centre, SA Pathology, Adelaide, SA, Australia.

${ }^{13}$ National Centre for Clinical Excellence on Emerging Drugs of Concern (NCCRED), National Drug and Alcohol Research Centre (NDARC), University of New South Wales, Sydney, Australia. ${ }^{14}$ Department of Infectious Diseases, Royal Brisbane and Women's Hospital, School of Medicine, University of Queensland, Brisbane, QLD, Australia. ${ }^{15}$ Department of Infectious Diseases and Microbiology, New South Wales Health Pathology, Royal Prince Alfred Hospital, Sydney, NSW, Australia. ${ }^{16}$ Department of Infectious Diseases, Peter MacCallum Cancer Centre, National Centre for Infections in Cancer, Melbourne, VIC, Australia.

Received: 23 January 2019 Accepted: 2 May 2019

Published online: 21 May 2019

\section{References}

1. Chapman B, Slavin M, Marriott D, Halliday C, Kidd S, Arthur I, et al. Changing epidemiology of candidaemia in Australia. J Antimicrob Chemother. 2017; 72(4):1103-8.

2. Kofteridis DP, Valachis A, Dimopoulou D, Andrianaki AM, Christidou A, Maraki S, et al. Factors influencing non-albicans Candidemia: a case-casecontrol study. Mycopathologia. 2017;182(7-8):665-72.

3. Magill SS, Edwards JR, Bamberg W, Beldavs ZG, Dumyati G, Kainer MA, et al. Multistate point-prevalence survey of health care-associated infections. N Engl J Med. 2014;370(13):1198-208.

4. Lockhart SR, lqbal N, Cleveland AA, Farley MM, Harrison LH, Bolden CB, et al. Species identification and antifungal susceptibility testing of Candida bloodstream isolates from population-based surveillance studies in two U.S. cities from 2008 to 2011. J Clin Microbiol. 2012;50(11):3435-42. 
5. Rajendran R, Sherry L, Deshpande A, Johnson EM, Hanson MF, Williams C, et al. A prospective surveillance study of Candidaemia: epidemiology, risk factors, antifungal treatment and outcome in hospitalized patients. Front Microbiol. 2016;7:915.

6. Chen S, Slavin M, Nguyen Q, Marriott D, Playford EG, Ellis D, et al. Active surveillance for candidemia, Australia. Emerg Infect Dis. 2006; 12(10):1508-16.

7. Andes DR, Safdar N, Baddley JW, Alexander B, Brumble L, Freifeld A, et al. The epidemiology and outcomes of invasive Candida infections among organ transplant recipients in the United States: results of the transplantassociated infection surveillance network (TRANSNET). Transpl Infect Dis. 2016;18(6):921-31.

8. Barchiesi F, Orsetti E, Mazzanti S, Trave F, Salvi A, Nitti C, et al. Candidemia in the elderly: what does it change? PLoS One. 2017;12(5):e0176576.

9. Puig-Asensio M, Fernandez-Ruiz M, Aguado JM, Merino P, Lora-Pablos D, Guinea J, et al. Propensity score analysis of the role of initial antifungal therapy in the outcome of Candida glabrata bloodstream infections. Antimicrob Agents Chemother. 2016;60(6):3291-300.

10. Marriott DJ, Playford EG, Chen S, Slavin M, Nguyen Q, Ellis D, et al. Determinants of mortality in non-neutropenic ICU patients with candidaemia. Crit Care. 2009;13(4):R115.

11. Playford EG, Lipman J, Jones M, Lau AF, Kabir M, Chen SC, et al. Problematic dichotomization of risk for intensive care unit (ICU)-acquired invasive candidiasis: results using a risk-predictive model to categorize 3 levels of risk from a multicenter prospective cohort of Australian ICU patients. Clin Infect Dis. 2016;63(11):1463-9.

12. Kollef M, Micek S, Hampton N, Doherty JA, Kumar A. Septic shock attributed to Candida infection: importance of empiric therapy and source control. Clin Infect Dis. 2012;54(12):1739-46.

13. Morrell M, Fraser VJ, Kollef MH. Delaying the empiric treatment of candida bloodstream infection until positive blood culture results are obtained: a potential risk factor for hospital mortality. Antimicrob Agents Chemother. 2005;49(9):3640-5.

14. Ostrosky-Zeichner L, Pappas PG, Shoham S, Reboli A, Barron MA, Sims $C$, et al. Improvement of a clinical prediction rule for clinical trials on prophylaxis for invasive candidiasis in the intensive care unit. Mycoses. 2011;54(1):46-51.

15. Chen SC, Sorrell TC, Chang CC, Paige EK, Bryant PA, Slavin MA. Consensus guidelines for the treatment of yeast infections in the haematology, oncology and intensive care setting, 2014. Intern Med J. 2014;44(12b):1315-32.

16. Pappas PG, Kauffman CA, Andes DR, Clancy CJ, Marr KA, OstroskyZeichner $L$, et al. Clinical practice guideline for the management of Candidiasis: 2016 update by the Infectious Diseases Society of America. Clin Infect Dis. 2016;62(4):e1-50.

17. Burrell AR, McLaws ML, Fullick M, Sullivan RB, Sindhusake D. SEPSIS KILLS: early intervention saves lives. Med J Aust. 2016;204(2):73 e1-7.

18. Li JS, Sexton DJ, Mick N, Nettles R, Fowler VG Jr, Ryan T, et al. Proposed modifications to the Duke criteria for the diagnosis of infective endocarditis. Clin Infect Dis. 2000;30(4):633-8.

19. White T, Bruns T, Lee $S$, et al. Amplification and direct sequencing of fungal ribosomal RNA genes for phylogenetics. In: Innis M, Gelfand D, Sninsky J, et al., editors. PCR protocols: a guide to methods and applications. New York: Academic Press; 1990. p. 315-22.

20. Clinical Laboratory Stadards Institute (CLSI). Reference method for broth dilution antifungal susceptibility testing of yeasts; fourth informational supplement M27-S4. Wayne, PA: CLSI; 2012.

21. Clinical Laboratory Stadards Institute (CLSI) Epidemiological Cutoff Values for Antifungal Susceptibility Testing - First edition; Approved standard M59. Wayne, PA, USA; 2016.

22. Li D, Xia R, Zhang Q, Bai C, Li Z, Zhang P. Evaluation of candidemia in epidemiology and risk factors among cancer patients in a cancer center of China: an 8-year case-control study. BMC Infect Dis. 2017;17(1):536.

23. Puig-Asensio M, Padilla B, Garnacho-Montero J, Zaragoza O, Aguado JM, Zaragoza R, et al. Epidemiology and predictive factors for early and late mortality in Candida bloodstream infections: a population-based surveillance in Spain. Clin Microbiol Infect. 2014;20(4):O245-54.

24. Ang BS, Telenti A, King B, Steckelberg JM, Wilson WR. Candidemia from a urinary tract source: microbiological aspects and clinical significance. Clin Infect Dis. 1993;17(4):662-6.

25. Fisher JF, Kavanagh K, Sobel JD, Kauffman CA, Newman CA. Candida urinary tract infection: pathogenesis. Clin Infect Dis. 2011;52(Suppl 6):S437-51.
26. Trubiano JA, Leung VK, Worth LJ, Teh BW, Thursky KA, Slavin MA. Candida glabrata fungaemia at an Australian cancer Centre: epidemiology, risk factors and therapy. Leuk Lymphoma. 2015;56(12):3442-4.

27. Grayson ML, Stewardson AJ, Russo PL, Ryan KE, Olsen KL, Havers SM, et al. Effects of the Australian National Hand Hygiene Initiative after 8 years on infection control practices, health-care worker education, and clinical outcomes: a longitudinal study. Lancet Infect Dis. 2018;18(11):1269-77.

28. Rosenthal VD, Maki DG, Graves N. The international nosocomial infection control consortium (INICC): goals and objectives, description of surveillance methods, and operational activities. Am J Infect Control. 2008;36(9):e1-12.

29. Khatib R, Johnson LB, Fakih MG, Riederer K, Briski L. Current trends in candidemia and species distribution among adults: Candida glabrata surpasses $\mathrm{C}$. albicans in diabetic patients and abdominal sources. Mycoses. 2016;59(12):781-6.

30. Cohen Y, Karoubi P, Adrie C, Gauzit R, Marsepoil T, Zarka D, et al. Early prediction of Candida glabrata fungemia in nonneutropenic critically ill patients. Crit Care Med. 2010;38(3):826-30.

31. Kristof K, Madach K, Sandor N, Ivanyi Z, Kiraly A, Erdei A, et al. Impact of molecular mimicry on the clinical course and outcome of sepsis syndrome. Mol Immunol. 2011;49(3):512-7.

32. Klingspor L, Tortorano AM, Peman J, Willinger B, Hamal P, Sendid B, et al. Invasive Candida infections in surgical patients in intensive care units: a prospective, multicentre survey initiated by the European Confederation of Medical Mycology (ECMM) (2006-2008). Clin Microbiol Infect. 2015;21(1):87 e1-e10.

33. Oude Lashof AM, Rothova A, Sobel JD, Ruhnke M, Pappas PG, Viscoli C, et al. Ocular manifestations of candidemia. Clin Infect Dis. 2011;53(3):262-8.

34. Seymour CW, Liu VX, Iwashyna TJ, Brunkhorst FM, Rea TD, Scherag A, et al. Assessment of clinical criteria for Sepsis: for the third international consensus definitions for Sepsis and septic shock (Sepsis-3). JAMA. 2016; 315(8):762-74

35. Simpson SQ. New Sepsis criteria: a change we should not make. Chest. 2016;149(5):1117-8.

36. National health and medical research council (NHMRC). Australian guidelines for the prevention and control of infection in healthcare. Commonwealth of Australia. 2010.

\section{Ready to submit your research? Choose BMC and benefit from:}

- fast, convenient online submission

- thorough peer review by experienced researchers in your field

- rapid publication on acceptance

- support for research data, including large and complex data types

- gold Open Access which fosters wider collaboration and increased citations

- maximum visibility for your research: over $100 \mathrm{M}$ website views per year

At BMC, research is always in progress.

Learn more biomedcentral.com/submissions 